\title{
Algunos problemas de docentes universitarios en la elaboración de artículos científicos
}

Some problems of university professors in preparing scientific articles

\author{
Volumen 17, Número 1 \\ Enero-Abril \\ pp. 1-17
}

\author{
Olga Gloria Barbón-Pérez \\ Ángela del Rocío Calderón-Tobar \\ Carlos Augusto Loza-Cevallos \\ Lenin Garcés-Viteri \\ Jorge Washington Fernández-Pino
}

Revista indizada en REDALYC, $\underline{\text { SCIELO }}$

Revista distribuida en las bases de datos:

LATINDEX, DOAJ, REDIB, IRESIE, CLASE, DIALNET, SHERPA/ROMEO, QUALIS-CAPES, MIAR

Revista registrada en los directorios:

ULRICH'S, REDIE, RINACE, OEI, MAESTROTECA, PREAL, CLACSO 


\title{
Algunos problemas de docentes universitarios en la elaboración de artículos científicos
}

Some problems of university professors in preparing scientific articles

\author{
Olga Gloria Barbón- Pérez \\ Ángela del Rocío Calderón-Tobar \\ Carlos Augusto Loza-Cevallos \\ Lenin Garcés-Viteri \\ Jorge Washington Fernández-Pino
}

Resumen: Actualmente, la mayor parte de las investigaciones sobre la producción científica de los docentes de la educación superior aborda el problema desde perspectivas ajenas a los procesos formativos universitarios, mediante los cuales estos sujetos desarrollan las habilidades y las competencias investigativas. Empleando un enfoque cuantitativo, se realizó un estudio observacional, descriptivo y transversal, orientado a presentar los principales resultados de la identificación, la categorización y la evolución de problemas de un grupo de docentes en la elaboración de artículos científicos. El referido a la discusión resultó ser el bloque de problemas que experimentó una menor disminución de la media entre revisiones. El bloque de problemas que experimentó una mayor disminución de la media entre revisiones fue la sección resultados. Constituyen tareas pendientes tanto el desarrollo de estrategias formativas para publicar sus trabajos científicos en las revistas académicas, como el reconocimiento del papel central que juegan los problemas en la elaboración de artículos científicos. Algunas recomendaciones que pudieran conllevar a que la población estudiada incremente su productividad científica apuntan a la toma de conciencia sobre la necesidad de construir una verdadera cultura científica en la comunidad universitaria, la desmitificación de la figura del científico como individuo poco común y genial, es decir, como un modelo casi inalcanzable, además de la promoción de la investigación colaborativa, la creación de estrategias individuales, grupales e institucionales para la publicación científica, la capacitación permanente y sistemática, un mayor estímulo por parte de los organismos que controlan la calidad de la educación a este nivel para aquellos investigadores noveles que logran publicar sus resultados, incluso en revistas de bajo impacto; entre otros.

Palabras clave: formación de docentes, estrategias educativas, comunicación científica, educación superior, docencia universitaria.

\begin{abstract}
Nowadays most of the research related to the scientific production of college teachers approach the problem from external perspectives related to college formative processes by which professors should develop investigative skills and competencies. Using a quantitative approach an observational, descriptive and crosssectional study was carried out. The study aims to present the main results of the identification, categorization and evolution of the problems of a group of professors in preparing scientific articles. Discussion turned out to be the block of problems which experienced a minor decrease in the average between reviews. The problem block that experienced a greater decrease in the mean between reviews was the results section. There are still pending tasks the development of training to publish their scientific papers in academic journals and the recognition of the central role of problems in the development of scientific papers strategies. Some recommendations that could lead to an increased scientific productivity in the population studied aims at raising awareness about the need to build a genuine scientific culture in the university community, demystifying scientists as rare and brilliant individuals, that is, as an almost unattainable model, also promoting collaborative research, creating individual strategies, group and institutional for scientific publication, ongoing and systematic training, further support by education quality control agencies for those young researchers who manage to publish their results, even in low impact journals; among others.
\end{abstract}

Key words: teacher training, educational strategies, scientific communication, higher education, academic teaching personnel.

\footnotetext{
${ }^{1}$ Los autores son Docentes Investigadores de la Universidad Nacional de Chimborazo, Ecuador.
}

Dirección electrónica de contacto: ogbarbon@unach.edu.ec

Artículo recibido: 3 de mayo, 2016

Enviado a corrección: 30 de junio, 2016

Aprobado: 21 de noviembre, 2016 


\section{Introducción}

El advenimiento del período actual de construcción de una sociedad del conocimiento ha acelerado los cambios que se experimentan hoy en la educación superior. Atrás ha quedado el tiempo cuando en las universidades latinoamericanas la intención de investigar quedaba en la retórica, a causa de sus deterioradas economías y problemas sociales que obligaban a centralizar los recursos en prioridades de naturaleza diferente.

El creciente interés por el tema en las últimas décadas ha dado origen a que en el contexto universitario se venga generando con mayor ímpetu la actividad investigativa, promovida por las exigencias de la sociedad a estas instituciones de educación superior, las cuales demandan orientar las acciones hacia el fortalecimiento, no solo de la docencia y de la extensión universitaria, sino también de la investigación, pues esta última representa un factor dinamizador de la búsqueda del saber y de la producción de nuevos conocimientos. En especial, es imprescindible lograr que los docentes universitarios se conviertan en permanentes investigadores de su labor educativa cotidiana, si se tiene en cuenta que cada día resulta más evidente el papel de la investigación como elemento esencial para el perfeccionamiento de la educación y "un componente esencial de la profesión académica" (Silvio, 2000, p. 15).

Esta realidad impone un reto a los procesos formativos universitarios mediante los cuales los docentes universitarios desarrollan las habilidades y las competencias que los dotan de herramientas investigativas y les permiten responder a las necesidades sociales de cada contexto (Barbón, López y Figueredo, 2014) y en ello radica la relevancia social de este estudio; el cual tiene como objetivo presentar los principales resultados de la identificación, la categorización y la evolución de los problemas de un grupo de docentes en la elaboración de artículos científicos.

\section{Breve referente teórico}

En concordancia con lo hasta aquí expuesto y ante estos desafíos, adquiere un rol protagónico la comunicación, componente fundamental de la investigación científica, pues ninguna investigación puede considerarse finalizada hasta que ha sido socializada en los foros habituales de la ciencia -congresos u otros eventos científicos, revistas académicas, libros, entre otros. En particular, la elaboración de artículos científicos es una de las actividades principales que desarrollan los miembros de las comunidades científicas 
contemporáneas (Cassany, López y Martí, 2000) y se reconoce como la primera forma de comunicar la ciencia entre las comunidades científicas (Mantilla et al., 2010).

Es preciso advertir que, aunque implica un importante compromiso cognitivo y de tiempo (Padilla y Carlino, 2010), y además supone que las investigaciones estén sujetas a revisión, evaluación y discusión de los diferentes grupos de científicos (Rogel, 2009); la divulgación de la ciencia en la forma de artículos científicos en revistas indexadas ha dejado de ser una opción y se ha transformado en una necesidad para los docentes universitarios. Esto se debe a que el aumento de las publicaciones indexadas juega un papel esencial, tanto en los procesos de asignación de recursos gubernamentales a los investigadores y a las instituciones, como en los procesos de acreditación de las instituciones de educación superior (Sabaj, 2009).

Este panorama da al traste con la imagen de las universidades y los colectivos docentes ajenos a la generación y promoción del conocimiento. De hecho, la publicación de artículos en revistas científicas indexadas es un componente de la evaluación de la actividad investigadora de los docentes universitarios, en este empeño constituye un modesto aporte la presente investigación. Los estudiosos de esta temática en la literatura especializada sugieren que uno de los principales frenos que se oponen al alcance de esta meta, amén de la indudable importancia antes resaltada que tiene la publicación en revistas académicas para este grupo de profesionales, es la llamativa de la ausencia de formación específica en este terreno. En efecto, los investigadores aprenden de manera informal a escribir y desarrollar estrategias para publicar sus trabajos científicos en las revistas académicas (Campanario, 2003).

Con respecto a esta problemática, en el contexto ecuatoriano, la formación de docentes-investigadores es reciente y en las universidades se observa una progresiva incorporación de programas de capacitación tendientes a propiciar el desarrollo de los conocimientos y de las habilidades de los docentes para la elaboración de artículos científicos. Es notable el avance logrado en la Universidad Nacional de Chimborazo (UNACH), la cual ha replanteado sus políticas en relación con las prácticas investigativas, permitiéndole avanzar significativamente en la gestión formativa de las competencias investigativas del personal académico, con el propósito de responder de forma eficiente y efectiva a los nuevos requerimientos emanados de los procesos de acreditación institucional. La Facultad de Ciencias de la Educación, Humanas y Tecnologías con los años ha ido ganando presencia en el escenario científico de la universidad, a partir del conjunto de actividades que desarrolla, hasta convertirse en un espejo del avance de las respectivas 
áreas de conocimiento. El incremento de proyectos de investigación y de publicaciones en revistas indexadas, así como la puesta en marcha de un sistema de talleres de capacitación en redacción y comunicación científica, son muestras significativas de que este enclave se sitúa a la vanguardia, consolidándose asimismo como un centro de referencia transformador, en su apuesta decidida por el fortalecimiento de la investigación científica en la UNACH y por el reconocimiento al esfuerzo realizado por los docentes-investigadores en este empeño.

No obstante, debido, entre otras causas, a que la mayor parte de las investigaciones relacionadas con la producción científica de los docentes universitarios aborda el problema desde una perspectiva sociológica, cienciométrica o administrativa (Luque, 2015; Vélez, Gómez, Úsuga y Vélez, 2014), escasea el enfoque pedagógico de la situación antes descrita, aspecto que constituye una de las fortalezas de este trabajo, dada su replicabilidad en otros contextos. En particular, no ha sido suficientemente investigado el rol central que juegan las insuficiencias en la elaboración de artículos científicos y su conexión con el origen de estas para plantear posibles soluciones. Por tal razón, se requiere precisar las dificultades del profesorado en la elaboración de artículos científicos con estudios que arrojen luz en cuanto a las limitaciones existentes en este sentido y favorezcan un mayor alcance de las alternativas formativas y de materiales específicos concebidos a partir de las necesidades detectadas.

\section{Metodología}

Entre mayo y agosto del 2015, se realizó un estudio observacional, descriptivo y transversal, a partir de un censo estadístico efectuado a una población de 44 docentes de la Facultad de Ciencias de la Educación, Humanas y Tecnologías de la Universidad Nacional de Chimborazo, matriculados en un sistema de talleres de capacitación en redacción y comunicación científica, los cuales se llevaron a cabo como parte de la estrategia institucional orientada hacia el fortalecimiento de la investigación científica. Los docentes, provenientes de diversas disciplinas y áreas de formación de la educación, las humanidades y las tecnologías, conformaban un grupo heterogéneo con respecto a sus niveles de preparación en redacción, en comunicación científica y en metodología de investigación.

El proceso investigativo atravesó por varias etapas; lo cual motivó que el estudio realizado se dividiera en cinco momentos esenciales que se describen seguidamente: fase de diseño, fase de formación, fase de identificación, fase de categorización, fase de estudio de la evolución de los problemas en la elaboración de artículos científicos. 
En la fase de diseño: se elaboró una propuesta conjunta y dialogada de sílabo y se gestionaron los recursos humanos y materiales así como se coordinaron las acciones para la ejecución del sistema de talleres. En estos, primaría el carácter participativo y sistémico, se realizarán exposiciones y debates teóricos mediante búsqueda parcial y conversación heurística y se favorecería la problematización y las aproximaciones sucesivas a los modelos de redacción científica.

En la fase de formación: se implementó un sistema de talleres de capacitación en redacción y comunicación científica, con enfoque constructivista, el cual consistió en 16 sesiones de cinco horas cada una, que se llevaron a cabo durante los meses de abril a agosto del 2015. Durante su desarrollo, los docentes estudiantes, supervisados por un claustro de 6 docentes-investigadores facilitadores, participaron activamente, redactaron, revisaron y corrigieron las partes estructurales de un artículo científico, para ello recibieron elementos de la teoría; conjuntamente con aspectos prácticos y fueron instruidos en la elaboración gradual de los artículos, apoyándose en un proceso tutorial presencial y virtual continuo, que posibilitó centrarse tanto en objetivos formativos de carácter más aplicado, como en el trabajo personalizado y en pequeños grupos, a fin de ofrecer orientaciones más específicas con vistas a la publicación científica en revistas indexadas, previamente seleccionadas, y desarrollar procesos de evaluación y monitoreo de las sucesivas reescrituras de los borradores de los artículos hasta la versión final.

En la fase de identificación: se elaboró un instrumento para la recogida de datos, fue la ficha de análisis documental, que tomó como referente la guía de autoevaluación de artículos científicos con formato IMRYD propuesta por Barbón (2015a). Este instrumento constó de 100 ítems y permitió obtener información tanto sobre las partes centrales, como las conexas de los artículos científicos (López, Alvarado y Mungaray, 2011). Se consideraron como partes centrales de un artículo: introducción, métodos, resultados, discusión y conclusiones. Las restantes se consideran como partes conexas (Cargill y O'Connor, 2009). Este instrumento fue sometido a un proceso de estudio de su confiabilidad y validez, administrándosele una prueba piloto a 10 docentes. Los resultados obtenidos fueron analizados, para garantizar la fiabilidad, mediante el cálculo del coeficiente de confiabilidad del alfa de Cronbach, el cual arrojó un valor de 0.8195. De igual manera, se realizó la validación de contenido de los ítems, utilizando la opinión de cinco expertos que laboran en el área de la redacción académica, la investigación y la publicación científica. Con base en sus recomendaciones, se efectuaron las correcciones pertinentes. 
En las fases de categorización y de estudio de la evolución de los problemas, los problemas de los docentes fueron clasificados en dos grandes grupos; problemas menores, entendiéndolos como aquellos que no motivarían el rechazo del trabajo por parte de la revista científica seleccionada para la publicación y problemas mayores, los cuales comprendían aquellos que darían al traste con la aceptación del trabajo (Barbón, 2015b). La evaluación de los trabajos se hizo teniendo en cuenta las instrucciones a los autores de la revista seleccionada por cada autor para enviar su manuscrito.

La toma de datos se efectuó directamente en las oficinas donde se recibieron los manuscritos. Es importante mencionar que los resultados que se presentan en este trabajo están en función de los registros de artículos en las bases de datos de la Dirección de Investigación de la Facultad de Ciencias de la Educación, Humanas y Tecnologías de la UNACH y que solo se tuvieron en cuenta aquellos artículos entregados en el período señalado y que se presentaron con la constancia de haber sido sujetos a, al menos, dos revisiones, la primera de ellas a cargo del claustro de docentes-investigadores, y la segunda, por parte del tutor correspondiente. Se procedió a la categorización por bloques de problemas, atendiendo a las partes de un artículo científico. La revisión se llevó a cabo por parte de los docentes-investigadores facilitadores de los talleres, de los cuales 5 ostentan el grado científico de doctorado, 2 son maestría, y al menos 2 poseen experiencia en los procesos de revisión y de arbitraje de artículos científicos en revistas indexadas. Estos desarrollaron una inestimable labor, contribuyendo de manera determinante, a la mejoría de la calidad científica de los manuscritos, a través de sus sugerencias, críticas y comentarios. Según los registros de la base de datos de la Dirección de Investigación de la Facultad, se revisaron un total de 52 manuscritos, de los cuales el $100 \%$ perteneció a los docentes que cursaban el sistema de talleres de capacitación en redacción y comunicación científica, resultando un promedio de 1.18 manuscritos por docente.

Los datos se tabularon mediante la confección de una base de datos de Excel con la información recolectada, la cual se procesó de forma automatizada utilizando el paquete estadístico SPSS en su versión 18.5 para Windows. En función de la naturaleza de las variables, se empleó la estadística descriptiva a partir del análisis de frecuencias y el cálculo porcentual, de manera que la información fue resumida mediante frecuencias absolutas y porcentajes para las variables cualitativas. Además se utilizaron medidas de tendencia central y de dispersión. A fin de proporcionar una medida más significativa sobre el punto hasta el cual se dispersan los problemas identificados alrededor de la media aritmética, se calculó la desviación estándar en cada bloque de problemas. Se estimaron los intervalos de 
confianza al $95 \%$ para completar la estimación puntual de las medidas mencionadas y el nivel de significación estadística se estableció en un valor de $p<0,05$.

\section{Resultados y su análisis}

A continuación se exponen los resultados que en primer lugar muestran que, en la primera revisión, los docentes presentaban fundamentalmente problemas mayores en la elaboración de los artículos científicos. De la totalidad de los mismos, solo 11 (25\%) presentaron trabajos que no serían rechazados por la revista científica seleccionada para su publicación. Un 75 \% de los docentes presentó trabajos que serían rechazados y, de ellos, un $54.5 \%$ presentó trabajos que incluían de 11 a 20 problemas incompatibles con la publicación científica. Ninguno entregó manuscritos con más de 20 problemas. En la segunda revisión, la mayoría de los docentes (61.3 \%) se agrupó entre los que lograron presentar trabajos con problemas menores. De los 17 docentes que aún en la segunda revisión entregaron trabajos que serían rechazados por las revistas, solo 10 (2.2\%) presentó trabajos que incluían de 11 a 20 problemas incompatibles con la publicación científica (Tabla 1).

Tabla 1

Relación entre la frecuencia de presentación y los grupos de problemas de los manuscritos entregados en la primera y segunda revisión

\begin{tabular}{|c|c|c|c|c|c|c|}
\hline \multirow{3}{*}{$\begin{array}{l}\text { Frecuencia de } \\
\text { presentación } \\
\text { de los problemas } \\
\text { por manuscrito }\end{array}$} & \multicolumn{3}{|c|}{$1^{\text {ra }}$ revisión } & \multicolumn{3}{|c|}{$2^{\text {da }}$ revisión } \\
\hline & \multicolumn{2}{|c|}{ N. ${ }^{\circ}$ de docentes $f(\%)$} & \multirow[t]{2}{*}{ Total } & \multicolumn{2}{|c|}{ N. ${ }^{\circ}$ de docentes $f(\%)$} & \multirow[t]{2}{*}{ Total } \\
\hline & $\begin{array}{l}\text { Problemas } \\
\text { menores }\end{array}$ & $\begin{array}{l}\text { Problemas } \\
\text { mayores }\end{array}$ & & $\begin{array}{l}\text { Problemas } \\
\text { menores }\end{array}$ & $\begin{array}{l}\text { Problemas } \\
\text { mayores }\end{array}$ & \\
\hline$<11$ & $2(4.5)$ & $9(20.4)$ & $11(25)$ & $11(25)$ & $7(15.9)$ & $\begin{array}{l}18 \\
(40.9)\end{array}$ \\
\hline $11-20$ & $9(20.4)$ & $24(54.5)$ & $33(75)$ & $16(36.3)$ & $10(2.2)$ & $\begin{array}{l}26 \\
(59.0)\end{array}$ \\
\hline$>20$ & - & - & & - & - & \\
\hline Total & $11(25)$ & $33(75)$ & $44(100)$ & $27(61.3)$ & 17 (38.6) & $44(100)$ \\
\hline
\end{tabular}

Como se aprecia en la tabla 2, al explorar la elaboración de la introducción de los artículos científicos, se constató que una gran mayoría (86.3\%) presentaba problemas en la redacción del objetivo, es decir, este no comenzaba con un verbo en infinitivo sino sustantivado. A este problema le siguió que no se exponía la relevancia, originalidad y contribución a nuevos conocimientos de la investigación realizada (29.5\%). En la segunda revisión, aunque la dificultad en la identificación de la posición del autor continúa, se aprecian cambios importantes, por ejemplo, el 100\% de los docentes logró eliminar los problemas referentes a la declaración del objetivo, la redacción de este y el manejo de las 
abreviaturas. Igualmente ocurrió un salto cualitativo en cuanto a la mención de la relevancia, originalidad y contribución a nuevos conocimientos de la investigación realizada. Los resultados de este segmento de problemas en la segunda revisión son más homogéneos con respecto a la media $(\mathrm{Dt}=11.1-5.3)$.

Tabla 2

Resultados de algunos problemas de los docentes en la elaboración de la introducción de los artículos científicos

\begin{tabular}{|l|l|l|c|}
\hline $\begin{array}{l}\text { Aspecto } \\
\text { evaluado }\end{array}$ & Problemas de los docentes & $\begin{array}{l}\mathbf{1}^{\text {ra }} \\
\text { revisión }\end{array}$ & $\begin{array}{c}\mathbf{2}^{\text {da }} \\
\text { revisión }\end{array}$ \\
\cline { 3 - 4 } & & $\mathbf{f}(\%)$ & $\mathbf{f}(\%)$ \\
\hline \multirow{3}{*}{ Objetivo } & No declara el objetivo de la investigación. & $8(18.1)$ & - \\
\cline { 2 - 4 } & $\begin{array}{l}\text { La redacción del objetivo no comienza con un verbo en } \\
\text { infinitivo. }\end{array}$ & $38(86.3)$ & $8(18.1)$ \\
\cline { 2 - 4 } & $\begin{array}{l}\text { No se utiliza un verbo que signifique una acción concreta } \\
\text { en la redacción del objetivo. }\end{array}$ & $3(6.8)$ & - \\
\cline { 2 - 4 } & No se encuadra dentro del propósito de la investigación. & $7(15.9)$ & - \\
\hline $\begin{array}{l}\text { Presentación } \\
\text { de } \\
\text { información }\end{array}$ & $\begin{array}{l}\text { Existen abreviaturas que no se describen la primera vez } \\
\text { que se mencionan. }\end{array}$ & $4(9)$ & - \\
\cline { 2 - 4 } & No se identifica la posición del autor. & $15(34)$ & $15(34)$ \\
\cline { 2 - 4 } & $\begin{array}{l}\text { No se expone la relevancia, originalidad y contribución a a } \\
\text { nuevos conocimientos de la investigación realizada. }\end{array}$ & $13(29.5)$ & $2(4.5)$ \\
\hline $\mathbf{m}$ & & 12.5 & 3.7 \\
\hline Dt & & 11.1 & 5.3 \\
\hline
\end{tabular}

Nota: f: frecuencia, m: media por bloque de problemas, Dt: desviación estándar.

Según se muestra en la Tabla 3, la media de problemas de 16.2 se modificó a 10.8 en la segunda revisión. La elaboración de la metodología de los artículos científicos constituyó un reto real para los docentes de este estudio, quienes en más de un $50 \%$ no consiguieron, en la primera revisión, describir el enfoque y metodología asumida en la investigación, la técnica de muestreo, o la población de origen incluyendo variables demográficas relevantes. Más del 50 \% de los sujetos no mencionó los criterios de inclusión y de exclusión ni presentó técnicas o índices adecuados de fiabilidad y validez de los instrumentos aplicados. Solo un $4.5 \%$ no describió los instrumentos utilizados para la recogida de datos. En la segunda revisión, el $100 \%$ de los docentes logró redactar la metodología en tiempo pretérito, así como indicar el lugar en el cual se realizó el estudio y describir los instrumentos de recolección de información. Con respecto a la primera revisión, permanecen tanto la falta de claridad en cuanto a los motivos que ocasionaron que se condujera el estudio de determinada forma, como que no se proporcionan datos descriptivos de la población de origen, incluyendo variables demográficas relevantes. En este bloque de problemas, el resto de los aspectos evaluados evidencian en mayor o menor grado, modificaciones positivas, con resultados de más variabilidad según la media ( $\mathrm{Dt}=7.1-8.1)$. 
Tabla 3

Resultados de algunos problemas de los docentes en la elaboración de la metodología de los artículos científicos

\begin{tabular}{|c|c|c|c|}
\hline \multirow{2}{*}{$\begin{array}{l}\text { Aspecto } \\
\text { evaluado }\end{array}$} & \multirow[t]{2}{*}{ Problemas de los docentes } & \multirow{2}{*}{$\frac{1^{\text {ra }} \text { revisión }}{f(\%)}$} & \multirow{2}{*}{$\frac{2^{\text {da }} \text { revisión }}{\text { f }(\%)}$} \\
\hline & & & \\
\hline \multirow[t]{4}{*}{ Diseño } & $\begin{array}{l}\text { El acápite de metodología no se redacta en tiempo } \\
\text { pretérito. }\end{array}$ & $11(25)$ & \\
\hline & $\begin{array}{l}\text { No se describe el enfoque y metodología asumida en la } \\
\text { investigación. }\end{array}$ & $24(54.5)$ & $8(18.1)$ \\
\hline & $\begin{array}{l}\text { No queda claro por qué se condujo el estudio de esta } \\
\text { manera. }\end{array}$ & $17(38.6)$ & $17(38.6)$ \\
\hline & No se indica el lugar donde se realizó el trabajo. & $18(40.9)$ & - \\
\hline \multirow{4}{*}{$\begin{array}{l}\text { Población } \\
\text { muestra }\end{array}$} & No se justifica la selección de variables ó de la muestra. & $20(45.4)$ & $18(40.9)$ \\
\hline & La muestra no es representativa y no se explica por qué. & $7(15.9)$ & $4(9)$ \\
\hline & $\begin{array}{l}\text { No se describe la técnica de muestreo, ni se mencionan } \\
\text { los criterios de inclusión y de exclusión. }\end{array}$ & $24(54.5)$ & $15(34)$ \\
\hline & $\begin{array}{l}\text { No se proporcionan datos descriptivos de la población de } \\
\text { origen incluyendo variables demográficas relevantes. }\end{array}$ & $24(54.5)$ & $24(54.5)$ \\
\hline \multirow[t]{3}{*}{ Instrumentos } & $\begin{array}{l}\text { No se describen los instrumentos utilizados para la } \\
\text { recogida de datos. }\end{array}$ & $2(4.5)$ & - \\
\hline & $\begin{array}{l}\text { No se identifican los métodos y los procedimientos } \\
\text { empleados. }\end{array}$ & $9(20.4)$ & $4(9)$ \\
\hline & $\begin{array}{l}\text { No se presentan técnicas e índices adecuados de } \\
\text { fiabilidad y validez de los instrumentos aplicados. }\end{array}$ & 24 (54.5) & $15(34)$ \\
\hline \multirow{2}{*}{$\begin{array}{l}\text { Procesamiento } \\
\text { matemático- } \\
\text { estadístico }\end{array}$} & $\begin{array}{l}\text { No se describen los métodos estadísticos con suficiente } \\
\text { detalle. }\end{array}$ & $20(45.4)$ & $18(40.9)$ \\
\hline & $\begin{array}{l}\text { No se presentan los resultados con los indicadores } \\
\text { apropiados de medición, error o intervalos de confianza. }\end{array}$ & $20(45.4)$ & $18(40.9)$ \\
\hline m & & 16.2 & 10.8 \\
\hline Dt & & 7.1 & 8.1 \\
\hline
\end{tabular}

Nota: f: frecuencia, m: media por bloque de problemas, Dt: desviación estándar.

En la Tabla 4 se reflejan los resultados de la valoración de la elaboración de los resultados de los artículos científicos por los sujetos de la investigación, se detectó que, en la primera revisión, con una media de 22.7, los principales problemas se relacionaban con que no aparecía la simbología explicativa al final de cada tabla (86.3\%), se utilizaban gráficas que duplicaban los datos contenidos en las tablas (75\%) y el título de cada tabla y figura no era suficientemente descriptivo (75\%). Llama la atención que para un $70.4 \%$ las variables o características no se acompañaban en la tabla o gráfica de una unidad de medida internacionalmente reconocida. Solo un 4.5 \% no logró presentar los hallazgos por orden de importancia. En la segunda revisión, aunque ocurren cambios muy leves en cuanto a la redacción del título de cada tabla y figura y el citar la fuente primaria al pie de la figura o la tabla, se observa cierta disminución de los problemas y una ligera variación de la homogeneidad con respecto a la media que logró contraerse a 12.7 (Dt=11.1-11.6). Es este el bloque de problemas que experimentó una mayor disminución de la media de problemas entre ambas revisiones. 
Tabla 4

Resultados de algunos problemas de los docentes en la elaboración de los resultados de los artículos científicos

\begin{tabular}{|c|c|c|c|}
\hline \multirow{2}{*}{$\begin{array}{l}\text { Aspecto } \\
\text { evaluado }\end{array}$} & \multirow[t]{2}{*}{ Problemas de los docentes } & $1^{\text {ra }}$ revisión & $2^{\text {da }}$ revisión \\
\hline & & $f(\%)$ & $f(\%)$ \\
\hline \multirow[t]{3}{*}{$\begin{array}{l}\text { Presentación del } \\
\text { contenido }\end{array}$} & $\begin{array}{l}\text { El acápite de resultados no se redacta en tiempo } \\
\text { pretérito. }\end{array}$ & $22(50)$ & $3(6.8)$ \\
\hline & $\begin{array}{l}\text { No se presentan los hallazgos por orden de } \\
\text { importancia. }\end{array}$ & $2(4.5)$ & 0 \\
\hline & Se incluye información innecesaria y repetitiva. & $22(50)$ & $7(15.9)$ \\
\hline \multirow[t]{8}{*}{ Tablas y figuras } & Las tablas y las figuras no se explican por sí solas. & $11(25)$ & 0 \\
\hline & $\begin{array}{l}\text { El título de cada tabla y figura no es suficientemente } \\
\text { descriptivo. }\end{array}$ & $33(75)$ & $25(56.8)$ \\
\hline & $\begin{array}{l}\text { Se cita la fuente primaria al pie de la figura o la } \\
\text { tabla. }\end{array}$ & $29(65.9)$ & $25(56.8)$ \\
\hline & $\begin{array}{l}\text { Las variables o características no se acompañan en } \\
\text { la tabla o gráfica de una unidad de medida } \\
\text { internacionalmente reconocida. }\end{array}$ & $31(70.4)$ & $23(52.2)$ \\
\hline & $\begin{array}{l}\text { Las tablas y figuras no siempre están referidas en el } \\
\text { texto o ubicadas lo más próximo posible a su } \\
\text { mención. }\end{array}$ & $22(50)$ & $3(6.8)$ \\
\hline & $\begin{array}{l}\text { Se utilizan gráficas que duplican los datos } \\
\text { contenidos en las tablas. }\end{array}$ & $33(75)$ & $24(54.5)$ \\
\hline & $\begin{array}{l}\text { No aparece la simbología explicativa al final de } \\
\text { cada tabla. }\end{array}$ & $38(86.3)$ & $29(65.9)$ \\
\hline & $\begin{array}{l}\text { Se fraccionan las tablas entre páginas y no se } \\
\text { repite el encabezado. }\end{array}$ & $7(15.9)$ & $1(2.2)$ \\
\hline $\mathbf{m}$ & & 22.7 & 12.7 \\
\hline Dt & & 11.1 & 11.6 \\
\hline
\end{tabular}

Nota: f: frecuencia, m: media por bloque de problemas, Dt: desviación estándar.

Con respecto a la elaboración de la discusión de los artículos científicos, la tabla 5 presenta resultados que indican que los docentes, con una media de problemas de 13.6 en la primera revisión, presentaron algunos problemas en este aspecto, a predominio de que repetían gran parte de los datos que ya habían sido reflejados en la sección Resultados y no mencionaban las limitaciones o fortalezas del estudio (56.8\%). Solo un $9 \%$ no contrastaba los resultados propios con aquellos obtenidos en trabajos previos. En cambio, en la segunda revisión, a pesar de que se mantienen los problemas en cuanto a que no se hace hincapié en los aspectos nuevos e importantes del estudio y las conclusiones que se derivan de ellos a la luz de la evidencia disponible, se repiten gran parte de los datos que ya han sido reflejados en la sección Resultados y no se mencionan las limitaciones o fortalezas del estudio, se aprecian transformaciones favorables en el resto de los aspectos y una mayor variabilidad de los datos con respecto a la media, que disminuyó a 10.8 (Dt=8.8-10.2) Es este el bloque de problemas que experimentó una menor disminución de la media de problemas entre ambas revisiones. 
Tabla 5

Resultados de algunos problemas de los docentes en la elaboración de la discusión de los artículos científicos

\begin{tabular}{|l|l|l|c|}
\hline $\begin{array}{l}\text { Aspecto } \\
\text { evaluado }\end{array}$ & Problemas de los docentes & $\mathbf{1}^{\text {ra }}$ revisión & $\mathbf{2}^{\text {da }}$ revisión \\
\cline { 2 - 4 } $\begin{array}{l}\text { Presentación } \\
\text { del contenido }\end{array}$ & $\begin{array}{l}\text { El acápite de discusión no se redacta en tiempo presente } \\
\text { al debatir y opinar sobre contenidos de otros autores y en } \\
\text { pasado cuando se abordan los resultados de una } \\
\text { investigación. }\end{array}$ & $14(31.8)$ & $4(9)$ \\
\cline { 2 - 4 } & $\begin{array}{l}\text { No se hace hincapié en los aspectos nuevos e importantes } \\
\text { del estudio y las conclusiones que se derivan de ellos a la } \\
\text { luz de la evidencia disponible. }\end{array}$ & $11(25)$ & $11(25)$ \\
\cline { 2 - 4 } & $\begin{array}{l}\text { Se repite gran parte de los datos que ya han sido } \\
\text { reflejados en la sección Resultados. }\end{array}$ & $25(56.8)$ & $24(54.5)$ \\
\cline { 2 - 4 } & No se mencionan las limitaciones o fortalezas del estudio. & $25(56.8)$ & $25(56.8)$ \\
\cline { 2 - 4 } & $\begin{array}{l}\text { No se abordan posibles explicaciones respecto a los } \\
\text { resultados o sus implicaciones para futuras investigaciones } \\
\text { en el campo de estudio. }\end{array}$ & $3(6.8)$ & $1(2.2)$ \\
\cline { 2 - 4 } & $\begin{array}{l}\text { No se contrastan los propios con resultados obtenidos en } \\
\text { trabajos previos. }\end{array}$ & $4(9)$ & 0 \\
\hline $\mathbf{m}$ & & 13.6 & 10.8 \\
\hline Dt & & 8.8 & 10.2 \\
\hline
\end{tabular}

Nota: f: frecuencia, m: media por bloque de problemas, Dt: desviación estándar.

En la tabla 6 se muestra una mejoría importante de la media de problemas, la cual se redujo de 23.4 en la primera revisión a 15.4 en la segunda. Además, se analizan las frecuencias y porcentajes de los problemas de los docentes en la elaboración de las referencias bibliográficas de los artículos científicos. En ella se pueden apreciar de forma clara que, en la primera revisión, la mayor parte de los docentes presentó dificultades, el análisis realizado arrojó que los problemas abarcan desde los referentes a que no siempre se utilizan fuentes de información de calidad tales como artículos científicos, libros, bases de datos, tesis, entrevistas y documentos normativos, hasta que la mayoría de las obras consultadas no son actuales, no se emplea una norma homogénea para el asentamiento bibliográfico, no todas las referencias se encontraban citadas apropiadamente dentro del texto o viceversa, no todas las referencias que aparecen en el texto figuran en la bibliografía. Solo un 15.9 \% presentó manuscritos en los cuales la mayoría de las citas no eran referenciales, sino textuales. En la segunda revisión, aunque persiste el problema de no emplear una norma homogénea para el asentamiento bibliográfico, se observa una clara disminución de los problemas y una mayor variabilidad con respecto a la media ( $\mathrm{Dt}=9.1$ 10.6). 
Tabla 6

Resultados de los principales problemas de los docentes en la elaboración de las referencias bibliográficas de los artículos científicos

\begin{tabular}{|l|l|l|c|}
\hline $\begin{array}{l}\text { Aspecto } \\
\text { evaluado }\end{array}$ & Problemas de los docentes & $\mathbf{1}^{\text {ra }}$ revisión & $\mathbf{2}^{\text {da }}$ revisión \\
\cline { 2 - 4 } $\begin{array}{l}\text { Calidad } \\
\text { actualidad }\end{array}$ & $\mathbf{f}(\%)$ & $\mathbf{f}(\%)$ \\
\hline & $\begin{array}{l}\text { No siempre se utilizan fuentes de información de } \\
\text { calidad, tales como artículos científicos, libros, } \\
\text { bases de datos, tesis, entrevistas y documentos } \\
\text { normativos. }\end{array}$ & $33(75)$ & $20(45.4)$ \\
\cline { 2 - 4 } $\begin{array}{l}\text { La mayoría de las obras consultadas no son } \\
\text { actuales. }\end{array}$ & $29(65.9)$ & $26(59)$ \\
\hline del contenido & $\begin{array}{l}\text { No se emplea una norma homogénea para el } \\
\text { asentamiento bibliográfico. }\end{array}$ & $33(75)$ & $33(75)$ \\
\cline { 2 - 4 } & $\begin{array}{l}\text { No todas las referencias se encuentran citadas } \\
\text { apropiadamente dentro del texto. }\end{array}$ & $22(50)$ & $11(25)$ \\
\cline { 2 - 4 } & $\begin{array}{l}\text { No todas las referencias que aparecen en el texto } \\
\text { figuran en la bibliografía. }\end{array}$ & $26(59)$ & $11(25)$ \\
\cline { 2 - 4 } & La mayoría de las citas no son referenciales. & $7(15.9)$ & 0 \\
\cline { 2 - 4 } & $\begin{array}{l}\text { El orden de los autores, sus nombres, la revista o } \\
\text { el hipervínculo no contienen la información } \\
\text { correcta. }\end{array}$ & $14(31.8)$ & $7(15.9)$ \\
\hline $\mathbf{m}$ & & 23.4 & 15.4 \\
\hline Dt & & 9.1 & 10.6 \\
\hline
\end{tabular}

Nota: f: frecuencia, m: media por bloque de problemas, Dt: desviación estándar.

En la actualidad, se asegura que la publicación científica se ha transformado en una práctica clave en la divulgación del conocimiento científico (Sierra, Buela, Paz y Santos, 2008) y un elemento importante especialmente en el proceso de transferencia de la información científica (Martinsson, 1983). No obstante, en el medio académico es común encontrar que la redacción se convierte en un problema para el investigador, cuando este intenta publicar sus trabajos en medios especializados. La dificultad que entraña el ejercicio de poner por escrito eficientemente lo que se ha investigado en ocasiones frustra al investigador y retrasa la difusión del conocimiento. Aparentemente un elevado número de investigadores comparte este punto de vista. (Cisneros y Olavy, 2012)

Un resultado que llama la atención en este estudio es cómo la elaboración de la metodología de los artículos científicos constituyó un reto real para los docentes, quienes, en más de un $50 \%$, no consiguieron, en la primera revisión, describir el enfoque y metodología asumida en la investigación, la técnica de muestreo, o la población de origen incluyendo variables demográficas relevantes. Esto puede deberse a que, según se menciona en la literatura revisada relativa al comportamiento de la elaboración de artículos científicos en universidades latinoamericanas de países como Argentina, Bolivia, Chile, Colombia, Costa Rica, Ecuador, México, Perú, Uruguay y Venezuela, en la gran mayoría de estos países se 
confirmó que la escritura de artículos científicos es considerada como un contenido que forma parte de la metodología de la investigación como materia de estudio, aunque la mayoría de las veces no se le aborde exhaustivamente o como una unidad temática independiente (Fondón, Madero y Sarmiento, 2010; Laufer, 2008).

Cabe resaltar que la indagación orientada hacia la identificación, la categorización y la evolución de algunos problemas de un grupo de docentes de la Facultad de Ciencias de la Educación, Humanas y Tecnologías de la UNACH en la elaboración de artículos científicos es pertinente y novedoso en este contexto. Aunque se han realizado algunos estudios sobre la productividad científica de las universidades, se trata de estudios generales por universidad y no son específicos por especialidades o por bloques de problemas que se oponen al incremento de la productividad científica de diversos grupos de docentes. (Buela, 2005; Sierra, Buela, Paz y Santos, 2009a; Campos, 2015)

Estableciendo un análisis comparativo con estudios afines realizados por otros autores, algunos estudios empíricos han documentado una serie de dificultades que enfrentan los sujetos que deben escribir un artículo científico. Datos similares a los anteriormente reseñados se obtuvieron en trabajos realizados por Jerz (2000), investigador que presenta cinco problemas típicos que enfrentan estudiantes de pregrado en la escritura técnica: a) la falta de revisión, b) el mal manejo del espacio en blanco, c) inconsistencia en el uso de siglas y abreviaciones, d) escasa jerarquización de la información y e) la "sobreventa" o "subventa" de contenidos (aspectos que se prometen y no se cumplen o viceversa). Sin embargo, para futuras investigaciones, se sugiere explorar este tipo de estudio con diferentes sujetos, sería factible realizarlo con docentes, pues la docencia universitaria tiene, como una actividad intrínsecamente relacionada, la investigación.

La enseñanza de la publicación científica abarca desde los elementos técnicos de cómo escribir correctamente un manuscrito, hasta las implicaciones éticas de la investigación o del contenido científico del trabajo. En trabajos de publicación más reciente, se encuentran algunos que en forma directa o indirecta corroboran esta idea (Franco y Rodríguez 2010; Sierra, Buela, Paz y Santos, 2009b). De cara al futuro se sugiere un mayor aprovechamiento del taller como forma de organización de la enseñanza, para el desarrollo y fortalecimiento de las habilidades de los docentes en la elaboración de artículos científicos, en tanto el mismo permite enfrentar actividades que, tal como ha sido estudiado por Restrepo (2012), resultan vitales, estas van desde promover el mantenimiento de ritmos de trabajo similares entre los participantes hasta verificar, atender dudas y evaluar los avances de los participantes. 
En el presente estudio, el referido a la Discusión resultó ser el bloque de problemas que experimentó una menor disminución de la media de problemas entre ambas revisiones. Frente a ello, el bloque de problemas que experimentó una mayor disminución de la media de problemas entre ambas revisiones fuera el perteneciente a la sección Resultados. Estos hallazgos son congruentes con la complejidad de este acápite, que, como todo proceso de escritura académica, en la secuencia didáctica, progresa desde la búsqueda de información, a la producción de un texto en un género que incluye el manejo de las modalidades discursivas expositiva y argumentativa. Lo cual implica el dominio de un saber y un saber hacer, que moviliza en el sujeto procesos metacognitivos, capacitándolo para resolver problemas concretos de diferentes grados de complejidad, según han mostrado otros estudios (Figueroa y Aillon, 2015; The University of Essex, 2012).

Una de las limitaciones de este estudio se vincula con el período cuando se realizó la recopilación de datos, que se prolongó desde mayo hasta agosto del 2015, con lo cual aquellos artículos que fueran entregados con posterioridad no pudieron ser analizados. Para una correcta interpretación de los resultados aquí expuestos hay que tener presente un sesgo importante; los problemas encontrados se hallaban condicionados por las categorías a evaluar en el instrumento aplicado. Otro sesgo que se reconoce es que el tiempo entre las revisiones no fue el mismo para todos los sujetos, respetando ritmos de aprendizaje y de trabajo heterogéneos, se decidió fijar más bien una fecha tope y no una fecha específica de entrega de los manuscritos.

\section{Conclusiones}

Se han presentado los resultados de la identificación, la categorización y la evolución de algunos problemas de un grupo de docentes en la elaboración de artículos científicos. De estas ideas se deriva como conclusión importante, primeramente, que la elaboración de un artículo científico es un proceso de aproximación y enriquecimiento sucesivo; en segundo lugar, se confirma la idea de que las revisiones de los trabajos, son efectivas, de gran utilidad para su calidad científica final, lo cual habla a favor de generalizar y sistematizar experiencias formativas similares; y en tercer lugar que aunque se ha avanzado en la estimulación, el apoyo y la creación de redes de formación científica de los docentes, constituyen tareas pendientes; por una parte, el desarrollo de estrategias formativas para publicar sus trabajos científicos en las revistas académicas y; por otra, el reconocimiento del papel central que juegan los problemas y su origen en la elaboración de artículos científicos, 
a fin de arrojar luz en cuanto a las limitaciones existentes en este sentido y favorecer un mayor alcance de las alternativas formativas.

No obstante, luego de señalar los inconvenientes que tributan a la escasa producción científica de los docentes de la educación superior, es preciso hacer referencia a las recomendaciones que pudieran conllevar a que esta población revierta dicha problemática incrementando su productividad científica. Entre los elementos que pudieran señalarse en esta dirección se encuentran: la toma de conciencia sobre la necesidad de construir una verdadera cultura científica en la comunidad universitaria, la desmitificación de la figura del científico como individuo poco común, genial; es decir, como un modelo casi inalcanzable, además de la promoción de la investigación colaborativa, la creación de estrategias individuales, grupales e institucionales para la publicación científica, la capacitación permanente y sistemática, un mayor estímulo por parte de los organismos que controlan la calidad de la educación a este nivel para aquellos investigadores noveles que logran publicar sus resultados, incluso en revistas de bajo impacto, la creación incentivos y de espacios de reconocimiento a aquellas revistas científicas seriadas que aún no alcanzan la indexación en bases de datos reconocidas y a los artículos que en ellas se publican; entre otros.

\section{Referencias}

Barbón Pérez, Olga Gloria, López Granda, Carlos y Figueredo Alarcón, Danisleydis. (2014). Cinco saberes para la formación de la competencia científico-investigativa con enfoque de profesionalización pedagógica. Revista Cubana de Reumatología, 16(2). Recuperado http://www.revreumatologia.sld.cu/index.php/reumatologia/article/view/333

Barbón Pérez, Olga Gloria. (2015a). Guía de 100 preguntas para la autoevaluación de artículos científicos con formato IMRYD. Material de apoyo a la investigación. Riobamba: Vicerrectorado de Investigación y Postgrado UNACH.

Barbón Pérez, Olga Gloria. (2015b). Algoritmo de clasificación de los problemas de los docentes en la elaboración de artículos científicos. Material de apoyo a la investigación. Riobamba: Vicerrectorado de Investigación y Postgrado UNACH.

Buela Casal, Gualberto. (2005). Situación actual de la productividad científica de las universidades españolas, International Journal of Clinical and Health Psychology, 5(1), 175-190. Recuperado de http://hdl.handle.net/10481/32653

Campanario, Juan Miguel. (2003). Cómo escribir y publicar un artículo científico. Cómo estudiar y aumentar su impacto. Rev.Esp.Doc.Cient, 26(4), 461-463, Recuperado de http://redc.revistas.csic.es/index.php/redc/article/viewArticle/203 
Campos Saborío, Natalia. (2015). ¿Por qué es importante que el personal docente investigue? Reflexión a partir de datos de una experiencia de investigación etnográfica. Revista Electrónica Actualidades Investigativas en Educación, 15(3), 1-16. DOI: http://dx.doi.org/10.15517/aie.v15i3.21071

Cargill, Margaret y O'Connor, Patrick. (2009). Writing Scientific Research Articles. Strategy and Steps. West Sussex: WileyBlackwell.

Cassany, Daniel, López Ferrero, Carmen y Martí, Jaume. (2000). Divulgación del discurso científico: La transformación de redes conceptuales. Hipótesis, modelo y estrategias. Discurso y sociedad, 2(2), 73-103.

Cisneros Estupiñan, Mireya y Olavy Arias, Giohanny. (2012). Alfabetización académica y lectura inferencial. Bogotá: Ed. Ecoe.

Figueroa, Beatriz y Aillon, Mariana. (2015). Escritura académica de un ensayo mediado por el aprendizaje colaborativo virtual. Estud. Pedagóg, 41(1). Recuperado de http://www.scielo.cl/scielo.php?script=sci arttext\&pid=S0718$\underline{07052015000100005 \& \operatorname{lng}=\mathrm{es} \& \mathrm{nrm}=\text { iso }}$

Fondón, Irene, Madero, María José y Sarmiento, Auxiliadora. (2010). Principales problemas de los profesores principiantes en la enseñanza universitaria. Formación universitaria, 3(2), 21-28. Recuperado de http://www.scielo.cl/scielo.php?script=sci arttext\&pid=S0718-50062010000200004

Franco, Carolina y Rodríguez Morales, Alfonso. (2010). Errores comunes en la redacción científica estudiantil. Gac Méd Caracas, 118(1), 69-72. Recuperado de http://www.scielo.org.ve/scielo.php?script=sci arttext\&pid=S0367$\underline{47622010000100011 \& \operatorname{lng}=\mathrm{es}}$

Jerz, Dennis. (2000). Top five problems. Writing. Recuperado de http://jerz.setonhill.edu/writing/technical/resume/tips.htm

Laufer, Miguel. (2008). Enseñar cómo publicar. Interciencia, 33(5), 321.

López Leyva, Santos, Alvarado Borrego, Aida y Mungaray Moctezuma, Ana Bárbara. (2011). La comunicación de la ciencia a través de artículos científicos. México: Juan Pablos Editores y U de O.

Luque Martínez, Teodoro. (2015). Actividad investigadora y contexto económico. El caso de las universidades públicas españolas. Revista Española de Documentación Científica, 38(1), e076. Recuperado de http://dx.doi.org/10.3989/redc.2015.1.1135

Mantilla Villarreal, Andrea, Medina Fontalvo, Jorge, Velasco Bayuelo, Carlos, Algarín Gregory, Jorge, Rodelo Salcedo, Eduardo, De la Rosa Barranco, Dereck y Caballero Uribe, Carlo. (2010). Guía práctica para publicar un artículo en revistas latinoamericana. Salud Uninorte, 26(2), 311-324. Recuperado de http://www.redalyc.org/articulo.oa?id=81719006012 
Martinsson, Anders. (1983). Guía para la redacción de artículos científicos destinados a la publicación ( $2^{\mathrm{da}}$ ed.). París: Unesco.

Padilla, Constanza y Carlino, Paula. (2010). Alfabetización académica e investigación acción: Enseñar a elaborar ponencias en la clase universitaria. En Giovanni Parodi (ed.), Alfabetización Académica y Profesional, Perspectivas contemporáneas (pp. 153-182). Santiago de Chile: Academia Chilena de la Lengua / Planeta.

Restrepo Baena, Emilio Alberto. (2012). Errores comunes en la elaboración de artículos científicos. Rev CES Med, 26(1), 131-134.

Rogel, Rosario. (2009). Apuntes del curso: "Elaboración de artículos científicos y criterios de arbitraje académico". Impartido por la Asociación Nacional de Instituciones de Educación Superior, Ciudad de México.

Sabaj, Omar. (2009). Descubriendo algunos problemas en la redacción de artículos de investigación científica (AIC) de alumnos de postgrado. Signos, 42(69), 107-127. Recuperado de http://www.scielo.cl/scielo.php?pid=S0718$09342009000100006 \&$ script $=$ sci abstract\&tlng=e

Sierra Freire, Juan Carlos, Buela Casal, Gualberto, Paz Bermúdez, María y Santos Iglesias, Pablo. (2008). Análisis transnacional del sistema de evaluación y selección del profesorado universitario. Interciencia, 33(4), 1-7.

Sierra Freire, Juan Carlos, Buela Casal, Gualberto, Paz Bermúdez, María y Santos Iglesias, Pablo. (2009a). Importancia de los criterios e indicadores de evaluación y acreditación del profesorado funcionario universitario en los distintos campos de conocimiento de la UNESCO. RUSC. Revista de Universidad y Sociedad del Conocimiento, 6(2), 23-32.

Sierra Freire, Juan Carlos, Buela Casal, Gualberto, Paz Bermúdez, María y Santos Iglesias, Pablo. (2009b). Diferencias por sexo en los criterios y estándares de productividad científica y docente en profesores funcionarios en España. Psicothema, 21(1), 124-132. Recuperado de: http://www.unioviedo.es/reunido/index.php/PST/article/view/8806/8670

Silvio Vaccarezza, Leonardo. (2000). Las estrategias de desempeño de la profesión académica. Ciencia periférica y sustentabilidad del rol de investigador universitario. Redes, 7(15), 15-43.

The University of Essex. (2012). The Department of Psychology Guide to Writing Research Reports. Department of Psychology. Recuperado de https://www.essex.ac.uk/psychology/documents/current/lab-reports.pdf

Vélez Cuartas, Gabriel, Gómez Flórez, Henry, Úsuga Ciro, Ana y Vélez Trujillo, Manuel. (2014). Diversidad y reconocimiento de la producción académica en los sistemas de evaluación de la investigación en Colombia. Revista Española de Documentación Científica, 37(3), e056. Recuperado de http://dx.doi.org/10.3989/redc.2014.3.1133 